\title{
ACCIONES DE DISEÑO EN CIMENTACIONES EN EDIFICIOS DE CONCRETO REFORZADO CON COMPORTAMIENTO INELÁSTICO
}

\author{
Luciano Roberto Fernández-Sola ${ }^{(1)}$, Luis Fernando Hernández-Rodríguez ${ }^{(2)}$, Carlos Ezequiel Loeza- \\ Pérez ${ }^{(2)}$ y Oscar Manuel González-Cuevas ${ }^{(1)}$
}

\begin{abstract}
RESUMEN
La capacidad de deformación inelástica es una propiedad fundamental de las estructuras cuando son sometidas ante las acciones de los sismos. En la actualidad, el diseño sismorresistente a nivel mundial se basa en asegurar que los sistemas estructurales tengan la capacidad de desarrollar las demandas de ductilidad tanto locales como globales, impuestas por las acciones máximas de diseño. Lograr que las estructuras incursionen en el intervalo de comportamiento inelástico de manera estable es el punto crítico. Para lograrlo, el sistema de cimentación debe ser capaz de transmitir las fuerzas que se desarrollan en la superestructura durante todo el proceso. En este trabajo se investigan las acciones máximas que la superestructura transmite a la cimentación a medida que desarrolla por completo su capacidad de deformación inelástica. Se utilizan los resultados de análisis estáticos no lineales en edificios de marcos de concreto reforzado como referencia. También se exploran algunas propuestas alternativas para poder determinar la magnitud de estas acciones sin la necesidad de desarrollar un análisis no lineal. Se muestra que la magnitud de las acciones máximas en la cimentación está íntimamente ligada con la sobrerresistencia del sistema.
\end{abstract}

Palabras clave: diseño por capacidad; diseño de cimentaciones; comportamiento inelástico

\section{DESIGN ACTIONS OVER FOUNDATIONS OF REINFORCED CONCRETE BUILDINGS WITH INELASTIC BEHAVIOR}

\begin{abstract}
The capacity of the structures to develop inelastic behavior is a fundamental property. Ensure the stability of the structure along all its inelastic behavior it's the most important issue on earthquake structural design, in order to provide the necessary inelastic capacity. The foundation must be able to transmit the forces produced on the superstructure up to the systems maximum inelastic displacement. In this paper, an analysis of the maximum forces produced by the superstructure over the foundation while developing all its inelastic capacity is presented. Push over analysis is used as reference method and only reinforced concrete frames are studied. Alternative simplified procedures are proposed to
\end{abstract}

\footnotetext{
Artículo recibido el 12 de abril de 2019 y aprobado para su publicación el 7 de diciembre de 2020. Se aceptarán comentarios y/o discusiones hasta cinco meses después de su publicación.

(1) Departamento de Materiales, Universidad Autónoma Metropolitana-Azcapotzalco, Av. San Pablo 180, Col. Reynosa Tamaulipas, Del. Azcapotzalco, Ciudad de México. C.P. 02200. 1rfs@azc.uam.mx; omgc@azc.uam.mx

(2) Posgrado en Ingeniería Estructural, Universidad Autónoma Metropolitana-Azcapotzalco, Av. San Pablo 180, Col. Reynosa Tamaulipas, Del. Azcapotzalco, Ciudad de México. C.P. 02200. ferdinandompg@gmail.com; loeza187@gmail.com
} 
avoid the necessity of non-linear analysis. It is shown that maximum forces introduced to foundation by the superstructure are associated with the structures overstrength ratio.

Keywords: capacity design, foundation design; inelastic behavior

\section{INTRODUCCIÓN}

Las prácticas actuales de diseño sismorresistente a nivel mundial se basan en los conceptos de comportamiento inelástico de las estructuras. Se ha estudiado ampliamente cuál es la secuencia de plastificación de los elementos estructurales adecuada para asegurar que el sistema estructural tenga un comportamiento inelástico estable y pueda desarrollar completamente su capacidad de deformación inelástica para diferentes estructuraciones. El uso del concepto del uso de fusibles estructurales ha sido ampliamente explotado e integrado en metodologías de diseño (Tena-Colunga y Ramírez, 2017, O’Reilly y Calvi, 2018) y de disipación de energía (Nangullasmú y Tena-Colunga, 2017, Nuzzo, et al. 2019, Lin, et al. 2019). En varios códigos de diseño alrededor del mundo, en ocasiones de manera implícita o en otras de manera explícita, los requerimientos de diseño dúctil buscan propiciar mecanismos de comportamiento inelástico deseados. Dichos mecanismos pueden promoverse por medio del uso de factores de reducción de resistencia diferidos para cada uno de los elementos y las comparaciones de resistencia de los elementos que confluyen en un mismo punto. Es necesario que la revisión de la secuencia de plastificación considere todas las partes del sistema estructural, incluido el sistema de cimentación. Para asegurar que el sistema estructural sea capaz de desarrollar completamente su capacidad de deformación inelástica, se requiere que el sistema suelo-cimentación permanezca elástico ante las acciones máximas que la superestructura pueda introducir en ella, durante todo el desarrollo del mecanismo de colapso.

De esta manera, el diseño sismorresistente actual considera que "toda estructura que es sometida a un terremoto intenso debería diseñarse para evitar el colapso más no para evitar sufrir daños estructurales" (Piqué del Pozo 2004). Se deben entonces identificar los niveles de daño esperado en la estructura para distintos niveles de solicitación, determinando un mecanismo de daño controlado de la estructura (Piqué del Pozo 2004). En general, dentro de este procedimiento de diseño no se especifican las consideraciones se deben tomar en cuenta para diseñar las cimentaciones.

Una manera de controlar el mecanismo de daño es mediante el diseño por capacidad, que permite controlar la manera en que la estructura incursiona en el intervalo de comportamiento inelástico (Palacios 2005, Burgos 2007, Castro 2005). Un ejemplo muy claro es el de un edificio a base marcos de acero contraventeados, donde el mecanismo que se espera para que la estructura permanezca estable a medida que desarrolla su capacidad inelástica, es el de columna fuerte-viga débil-contraviento más débil (Tapia y Tena 2013). En otras partes del mundo se han desarrollado ideas similares en las cuales se incluye la cimentación, tal es el caso de utilizar la falla del suelo para dar protección sísmica a las estructuras (Anastasopoulos et al. 2010).

La cimentación (sub-estructura) es una parte fundamental de la estructura cuya función principal es la de transmitir las cargas hacia el terreno. Por lo general, la determinación de las cargas provenientes de la superestructura son responsabilidad del ingeniero estructural. Considerando que se requiere que la estructura desarrolle por completo su capacidad, se tiene que saber con precisión las fuerzas máximas que se pueden producir en la sub-estructura para garantizar el comportamiento esperado.

Siguiendo la idea de los mecanismos de daño deseados, en este trabajo se utiliza el concepto de superestructura débil-cimentación fuerte, considerando la necesidad que el sistema suelo cimentación permanezca cuasi-elástico a medida que la superestructura desarrolla toda su capacidad. Consideremos una 
estructura representada por la curva de capacidad (cortante basal $V$ contra desplazamiento $\Delta$ ) mostrada en la figura 1. Siguiendo las metodologías actuales de diseño, la fuerza cortante de diseño reducida por comportamiento inelástico y sobrerresistencia está representada por el cortante $V_{d}$. Debido a las decisiones de diseño y las resistencias nominales de los materiales, la fuerza cortante para la cuál se presentará la primera fluencia en la estructura será mayor $\left(V_{y}\right)$. Adicionalmente, la redundancia de los sistemas estructurales y la redistribución de elementos mecánicos produce que la estructura incursione progresivamente en el intervalo de comportamiento inelástico, en la cuál la fuerza cortante deberá seguirse incrementando para producir mayores desplazamientos. El valor máximo de la fuerza cortante está asociado con el desplazamiento inelástico correspondiente a la demanda de ductilidad esperada en la estructura $\left(V_{u}\right)$. Considerando que la cimentación suele diseñarse utilizando las fuerzas actuantes de diseño ( $V_{d}$ en este caso) es probable que la resistencia de la cimentación no sea suficiente para asegurar que la estructura desarrolle toda su capacidad.

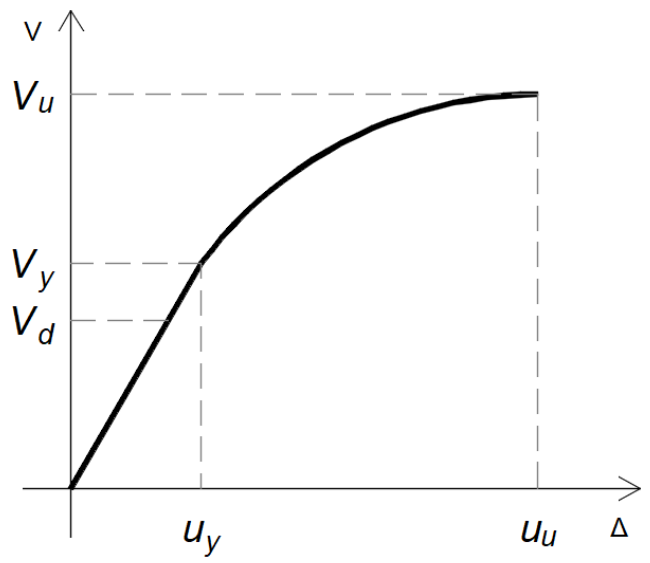

Figura 1. Curva de capacidad esquemática de una estructura redundante

La naturaleza de los mecanismos de falla de los sistemas suelo cimentación es compleja, dado que se debe de considerar la posibilidad de la falla de los elementos estructurales de la cimentación y de los diferentes tipos de falla en la masa de suelo. La redundancia de los sistemas estructurales de la cimentación es variable, y por lo tanto su sobrerresistencia. Respecto a los diversos tipos de falla de la masa del suelo, es probable que se desarrollen de manera progresiva, lo cual aunado a las grandes reducciones de resistencia que suelen utilizarse en el suelo, genera valores altos de sobrerresistencia en estos sistemas. Sin embargo, no se han determinado de manera explícita los valores de sobrerresistencia para los sistemas suelo cimentación. Dado que la práctica actual del diseño sismorresistente se inclina cada vez más a caracterizar e intentar controlar el comportamiento inelástico de los sistemas estructurales, es necesario comenzar a extender los conceptos del diseño por capacidad a los sistemas suelo cimentación, mediante la determinación de las acciones máximas probables que se pueden producir y los valores de la sobrerresistencia de los diversos componentes de dichos sistemas.

En este trabajo se dan recomendaciones de las acciones de diseño sobre la cimentación que deben considerarse para garantizar un comportamiento edificio fuerte-cimentación más fuerte y que el diseño por capacidad se cumpla de manera estable.

\section{DESCRIPCIÓN DE LAS ESTRUCTURAS ANALIZADAS}

Los edificios analizados están estructurados con marcos de concreto reforzado. Se consideran edificios de seis y diez niveles. Todos los edificios tienen la misma planta cuadrada de tres crujías en ambos 
sentidos mostrada en la figura 2. En todos los casos los sistemas de piso se modelaron como diafragma rígido en todos los niveles. En altura, todos los modelos tienen un entrepiso de $3.5 \mathrm{~m}$ en planta baja, y $3 \mathrm{~m}$ en los niveles subsecuentes. No se consideran irregularidades ni discontinuidades del edificio.

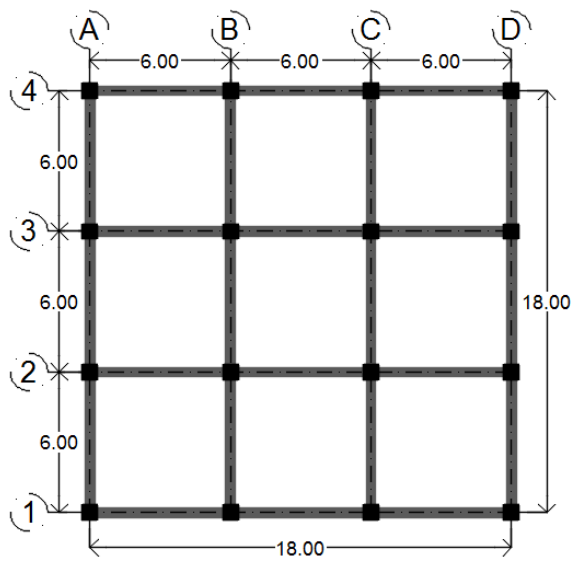

Figura 2. Planta de los edificios estudiados

Tabla 1. Secciones y armados longitudinales de los seis modelos

\begin{tabular}{|c|c|c|c|c|c|c|}
\hline Modelo & $6 B R Q 2$ & $6 B R Q 4$ & $10 B R Q 2$ & $10 B F Q 2$ & $10 B R Q 4$ & $10 B F Q 4$ \\
\hline$f^{\prime} c\left(\mathrm{kgf} / \mathrm{cm}^{2}\right)$ & 350 & 350 & 350 & 350 & 350 & 350 \\
\hline fy $\left(\mathrm{kgf} / \mathrm{cm}^{2}\right)$ & 4,200 & 4,200 & 4,200 & 4,200 & 4,200 & 4,200 \\
\hline \multirow{6}{*}{$\begin{array}{c}\text { Sección de columnas }(\mathrm{cm}) \\
\text { Niveles }\end{array}$} & \multirow{6}{*}{$\begin{array}{l}70 \times 70(\mathrm{C}) \\
60 \times 60(\mathrm{P}) \\
\quad 1 \text { al } 6\end{array}$} & $70 \times 70$ & $85 \times 85$ & $85 \times 85$ & $70 \times 70$ & $70 \times 70$ \\
\hline & & $1 y 2$ & $1 y 2$ & $1 y 2$ & $1 y 2$ & 1 y 2 \\
\hline & & $60 \times 60$ & $75 \times 75$ & $75 \times 75$ & $60 \times 60$ & $60 \times 60$ \\
\hline & & 3 al 5 & 3 al 7 & 3 al 7 & 3 al 7 & 3 al 7 \\
\hline & & $50 \times 50$ & $70 \times 70$ & $70 \times 70$ & $50 \times 50$ & $50 \times 50$ \\
\hline & & 6 & 8 al 10 & 8 al 10 & 8 al 10 & 8 al 10 \\
\hline \multirow{7}{*}{$\begin{array}{c}\text { Refuerzo longitudinal } \\
\text { (No de barras \#diámetro) } \\
\text { Niveles }\end{array}$} & $8 \# 10(C)$ & & & & \multirow{7}{*}{$\begin{array}{l}16 \# 8 \\
1 \text { al } 10\end{array}$} & \multirow{7}{*}{$\begin{array}{l}16 \# 8 \\
1 \text { al } 10\end{array}$} \\
\hline & $\begin{array}{c}11 \# 12(\mathrm{P}) \\
1 \text { y } 2\end{array}$ & 1 al 3 & $\begin{array}{l}32 \text { \# } 8 \\
1 \text { y } 2\end{array}$ & $\begin{array}{l}32 \text { \#8 } \\
1 \text { y } 2\end{array}$ & & \\
\hline & $8 \# 9(\mathrm{C})$ & \multirow{5}{*}{$\begin{array}{c}8 \# 7 \\
4 \text { al } 6\end{array}$} & $16 \# 8$ & $16 \# 8$ & & \\
\hline & $\begin{array}{c}8 \# 10(\mathrm{P}) \\
3 \text { y } 4\end{array}$ & & 3 al 7 & $\begin{array}{l}10 \text { ald } \\
3 \text { al }\end{array}$ & & \\
\hline & $8 \# 8(C)$ & & & & & \\
\hline & $8 \# 9(\mathrm{P})$ & & $16 \# 6$ & $16 \# 6$ & & \\
\hline & $5 y 6$ & & & & & \\
\hline \multirow{6}{*}{$\begin{array}{l}\text { Sección de vigas }(\mathrm{cm}) \\
\text { Niveles }\end{array}$} & & $35 \times 70$ & $55 \times 75$ & $55 \times 75$ & $45 \times 65$ & $45 \times 65$ \\
\hline & & 1 al 3 & 1 & 1 & 1 & 1 \\
\hline & $35 \times 70$ & \multirow{4}{*}{$\begin{array}{c}30 \times 60 \\
4 \text { al } 6\end{array}$} & $50 \times 75$ & $50 \times 75$ & $40 \times 65$ & $40 \times 65$ \\
\hline & 1 al 6 & & 2 al 7 & 2 al 7 & 2 al 7 & 2 al 7 \\
\hline & & & $45 \times 75$ & $45 \times 75$ & $35 \times 65$ & $35 \times 65$ \\
\hline & & & 8 al 10 & 8 al 10 & 8 al 10 & 8 al 10 \\
\hline \multirow{11}{*}{$\begin{array}{c}\text { Refuerzo longitudinal } \\
\text { (No de barras \#diámetro) } \\
\text { Niveles }\end{array}$} & $7 \# 8(s)$ & \multirow{6}{*}{$\begin{array}{l}5 \# 7(\mathrm{~s}) \\
2 \# 7(\mathrm{i}) \\
1 \text { al } 3\end{array}$} & 6\#8 (s) & 6\#8 (s) & 6\#6 (s) & 6\#6 (s) \\
\hline & 6\#8 (i) & & $9 \# 6$ (i) & 9\#6 (i) & 4\#6 (i) & 4\#6 (i) \\
\hline & $1 \mathrm{al} 3$ & & 1 & 1 & 1 & 1 \\
\hline & $5 \# 8(s)$ & & $7 \# 8(s)$ & $7 \# 8(s)$ & $7 \# 6(s)$ & $7 \# 6(s)$ \\
\hline & 4\#8 (i) & & $6 \# 8$ (i) & 6\#8 (i) & $5 \# 6$ (i) & $5 \# 6$ (i) \\
\hline & 4 & & 2 al 7 & 2 al 7 & 2 al 7 & 2 al 7 \\
\hline & $5 \# 8(s)$ & \multirow{5}{*}{$\begin{array}{l}5 \# 6(\mathrm{~s}) \\
2 \# 6(\mathrm{i}) \\
4 \text { al } 6\end{array}$} & \multirow{5}{*}{$\begin{array}{l}8 \# 6(s) \\
6 \# 6(\mathrm{i}) \\
8 \text { al } 10\end{array}$} & \multirow{5}{*}{$\begin{array}{l}8 \# 6(\mathrm{~s}) \\
6 \# 6(\mathrm{i}) \\
8 \text { al } 10\end{array}$} & \multirow{5}{*}{$\begin{array}{l}5 \# 6(\mathrm{~s}) \\
3 \# 6(\mathrm{i}) \\
8 \text { al } 10\end{array}$} & \multirow{5}{*}{$\begin{array}{l}5 \# 6(\mathrm{~s}) \\
3 \# 6(\mathrm{i}) \\
8 \text { al } 10\end{array}$} \\
\hline & 3\#8 (i) & & & & & \\
\hline & $\frac{5}{3 \# 8(s)}$ & & & & & \\
\hline & $\begin{array}{l}3 \# 8 \text { (s) } \\
3 \# 8 \text { (i) }\end{array}$ & & & & & \\
\hline & $\begin{array}{c}3 \# 8 \text { (i) } \\
6\end{array}$ & & & & & \\
\hline
\end{tabular}


Los edificios se analizaron y diseñaron de acuerdo con los procedimientos normativos incluidos en las normas técnicas complementarias del reglamento de construcciones de la Ciudad de México. Los modelos de seis niveles se analizaron y diseñaron considerando base rígida (sin efectos de interacción suelo estructura) de acuerdo con los procedimientos incluidos en las NTC-04. Los edificios de diez niveles se analizaron y diseñaron con base rígida y con base flexible considerando los efectos de interacción suelo estructura de acuerdo con los procedimientos incluidos en las NTC-17. Todos los edificios se diseñaron para ductilidad media $(Q=2)$ y para alta ductilidad $(Q=4)$. De esta manera se presentan resultados para seis modelos, dos de seis niveles ( $6 B R Q 2$ y $6 B R Q 4)$ y cuatro de diez niveles, dos sin efectos de interacción suelo estructura (10BRQ2 y 10BRQ4) y dos en base flexible considerando los efectos de interacción suelo estructura (10BFQ2 y $10 B F Q 4$ ). Adicionalmente, se presentan los resultados de AENL a los edificios de seis niveles con base flexible en comparación con los de base rígida. Los modelos con base flexible incorporan resortes traslacionales y rotacionales obtenidos de las funciones de impedancia calculadas de acuerdo con el procedimiento incluido en las NTC-17 que representan la deformación de la base.

Los modelos de seis niveles se ubicaron en un sitio con un periodo natural de vibrar $T_{s}=2.2 \mathrm{~s}$ (Zona IIIb de acuerdo con las NTC-04), con una profundidad del estrato compresible de $H_{s}=40 \mathrm{~m}$. Para el caso de los modelos de 10 niveles, se ubicaron en un sitio con un $T_{s}=1.2 \mathrm{~s}$ con una profundidad del estrato compresible de $H_{s}=22 \mathrm{~m}$. Las propiedades del suelo se tomaron de acuerdo con los valores sugeridos en las NTC-04 para la arcilla del valle de México $\left(\gamma=1.25 \mathrm{tonf} / \mathrm{m}^{3} ; \zeta=0.03\right.$ y $\left.v=0.45\right)$. En la tabla 1 se resumen las secciones y los armados de los modelos. El refuerzo transversal cumple con las condiciones establecidas en las NTC-04 y NTC-17 y no se reportan en este trabajo por cuestión de espacio. Los detalles de los diseños de los edificios de seis niveles pueden consultarse en Hernández-Rodríguez (2016) y los de los edificios de 10 niveles en Loeza-Pérez (2019).

En la tabla 2 se reportan los valores de los parámetros dinámicos de los modelos. Para el caso de los modelos con base flexible, los valores que se reportan son lo modificados por efectos de interacción suelo estructura de acuerdo con lo estipulado en las NTC-17. En el caso de las estructuras con base flexible, los valores del amortiguamiento del sistema se ven incrementados. De manera inversa, los valores de los factores de reducción de fuerzas sísmicas $Q$ ' son menores debidos a los efectos de interacción suelo estructura. Una explicación mas detallada de este efecto puede consultarse en Avilés y Pérez-Rocha (2003) y en Fernández-Sola y Huerta-Écatl (2018).

Tabla 2. Parámetros dinámicos de los seis modelos

\begin{tabular}{ccccccc}
\hline Modelo & $6 B R Q 2$ & $6 B R Q 4$ & $10 B R Q 2$ & $10 B F Q 2$ & $10 B R Q 4$ & $10 B F Q 4$ \\
\hline Periodo fundamental (s) & 0.84 & 0.87 & 0.98 & 1.56 & 1.25 & 1.7 \\
Amortiguamiento (\%) & 5 & 5 & 5 & 8.4 & 5 & 7.8 \\
$Q$ ' para el periodo fundamental & 2 & 4 & 2.12 & 1.38 & 4.35 & 2.63 \\
$R$ para el periodo fundamental & 2 & 2 & 2 & 2 & 2 & 2 \\
\hline
\end{tabular}

En los análisis estáticos no lineales (AENL) se utilizaron modelos de plasticidad concentrada en los extremos de las vigas y de las columnas. Las relaciones momento curvatura $(M-\varphi)$ de las secciones transversales se calcularon utilizando el programa BIAX (Wallace, 1992). Se verificó que las rotaciones plásticas calculadas con este programa de cómputo coincidieran con las rotaciones plásticas recomendadas por el FEMA-356. La longitud plástica se calcula como el peralte entre dos $(d / 2)$.

El programa BIAX utiliza el modelo constitutivo de Kent y Park modificado para el concreto. Este modelo toma en cuenta el confinamiento del concreto en el aumento de la resistencia. Por otra parte, para representar el modelo del acero se utilizaron las propiedades reportadas por Rodríguez y Botero (1996) para aceros mexicanos. 
En este trabajo se seleccionó un patrón de cargas laterales tipo triangular invertido para todos los modelos. La capacidad obtenida es dependiente del patrón de cargas seleccionado y el patrón utilizado es congruente con la respuesta de los edificios en donde su primer modo de vibración es dominante.

El análisis estático no lineal (AENL) se realizó en el programa Sap2000 (CSI, 2017), se realizaron dos estados de carga, uno que contempla las cargas gravitacionales existentes en la estructura y el segundo el que contiene el patrón de cargas laterales triangulares invertidas. Para ambos estados de carga se consideraron los efectos $\mathrm{P}-\Delta$, el programa se alimentó con los datos obtenidos por medio de los diagramas momento curvatura $M-\varphi$.

Se realizó un análisis previo calculando los diagramas momento curvatura de todas las columnas con la influencia de la carga axial derivada de la carga gravitacional. A partir de ese punto se tomaron las cargas axiales existentes para el primer paso del análisis estático no lineal, para uno intermedio y para el último paso. Posteriormente se calcularon los diagramas momento-curvatura para cada carga axial de cada paso y se alimentó de nuevo el programa Sap2000 (CSI, 2017), esto se hizo para tomar en cuenta la influencia de la carga axial en la respuesta, ya que algunas columnas están trabajando en flexo-tensión.

En los modelos que consideran base flexible (BF), la subestructura consiste en un cajón de cimentación. Dado que la definición de las funciones de impedancia considera que el sistema estructural de la cimentación es infinitamente rígido, se restringen todos los nodos que conforman el cajón mediante una restricción de cuerpo rígido referidos a un nodo maestro. En el nodo maestro se modela un juego de cuatro resortes (dos en traslación y dos en rotación) con los valores calculados de acuerdo con las NTCS-17. El nodo maestro se ubica en el centroide de la cimentación en planta y en la parte inferior de la misma, en consistencia con las hipótesis consideradas en la definición de las funciones de impedancia (Gazetas, 1991). En la figura 3 se muestra el modelado de los efectos ISE en los modelos de diez niveles. Los detalles de esta manera de modelar los efectos de interacción suelo estructura pueden consultarse en Fernández-Sola et al. (2017) y en Fernández-Sola y Huerta-Écatl (2018).
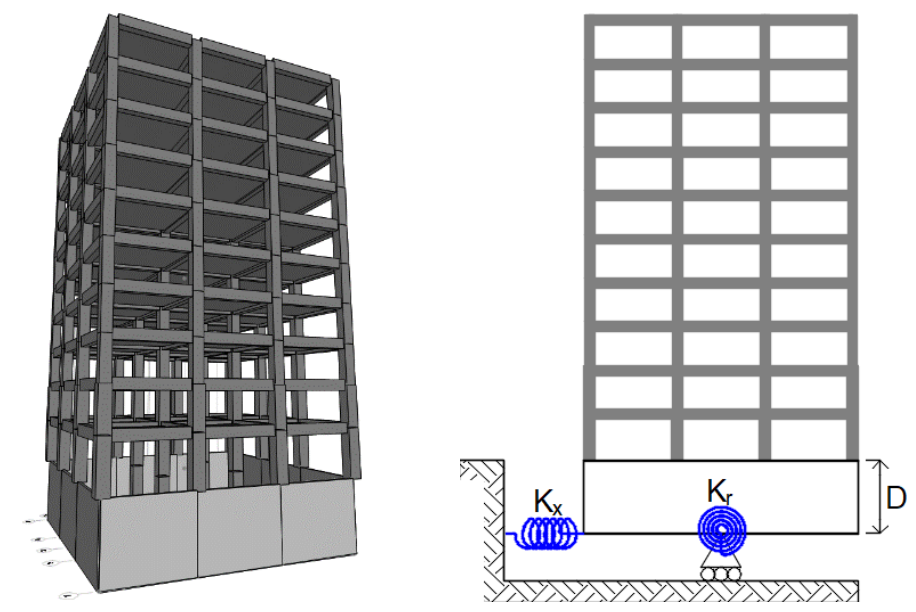

Figura 3. Esquema del modelado de la flexibilidad de la base

\section{COMPARACIÓN DE RESULTADOS}

Como ya se ha mencionado, es necesario reconocer que las acciones de diseño sobre los sistemas suelo cimentación deben considerar las cargas máximas que puede introducir la superestructura. En el caso 
de sistemas que estarán sujetos a demandas de ductilidad elevadas, estas acciones máximas deben estar definidas para los desplazamientos máximos que se considera se presentarán en las estructuras. Estas acciones serán mayores a las de diseño de la superestructura, debido a la sobrerresistencia que experimentan los diversos sistemas estructurales. Una forma para determinar estas acciones es mediante un Análisis Estático No Lineal (AENL) en el cuál se caractericen las acciones de los elementos estructurales de planta baja que se desarrollan para el desplazamiento máximo esperado de la estructura. Sin embargo, existen muchas ocasiones en las cuales es necesario facilitar a los diseñadores una aproximación de estas acciones sin tener que desarrollar AENL completos. En este trabajo se exploran diversas alternativas para el cálculo de las fuerzas cortantes y momentos flectores en la base de las columnas de planta baja. Se presentan cuatro alternativas asociadas a los resultados de los análisis modales espectrales utilizando espectros de diseño modificados. Se ha decidido utilizar espectros de diseño modificados para que los efectos asociados con la sobrerresistencia de los sistemas solamente influyan en las fuerzas asociadas con las acciones sísmicas. Estas modificaciones están referidas a los valores de pseudoaceleración elástica $(S a)$, el factor de sobrerresistencia $(R)$ y el factor de reducción de fuerzas sísmicas $\left(Q^{\prime}\right)$. Adicionalmente se incluyen los resultados utilizando la resistencia real de las columnas a flexión y la fuerza cortante asociada, siguiendo los requisitos del diseño por capacidad. A continuación, se detallan las alternativas correspondientes al uso de espectros de diseño modificados.

- AENL; con este criterio se obtienen los elementos mecánicos a partir de un análisis estático no lineal en el punto en el que el sistema estructural ya no es capaz de incrementar su cortante basal. Este análisis se toma como referencia dado que representa mejor el comportamiento esperado de la capacidad máxima de la estructura.

- $\quad S a / R Q$ '; estas fuerzas de diseño corresponden a los elementos mecánicos de diseño utilizados para el diseño de la superestrutcura considerando el comportamiento inelástico de la misma. Este criterio es el que utilizaban las Normas Técnicas Complementarias para Diseño y Construcción de Cimentaciones 2004 y algunos otros códigos en el mundo. En este criterio no se considera ningún concepto de diseño por capacidad ni la sobrerresistencia de la superestructura.

- $\quad S a$; estas fuerzas de diseño corresponden al comportamiento elástico de la superestructura.

- $\quad S a / R$; en este caso las fuerzas de diseño se obtendrán por medio del análisis utilizando el espectro cuya ordenada espectral se encuentra dividida entre $\mathrm{R}$ y considera un factor de comportamiento sísmico $\mathrm{Q}=1$. Este espectro corresponde al espectro elástico incluido en el cuerpo principal de las NTC-04.

- $\quad S a / Q^{\prime}$, esta consideración es equivalente al uso de las fuerzas de diseño multiplicadas por el factor de sobrerresistencia $R$. El uso de las fuerzas de diseño multiplicadas por $R$ es el criterio es el que se sugiere en varios códigos de diseño.

- $\quad$ NTCS-17, en estos resultados se utilizan los elementos mecánicos obtenidos del caso $S a / R Q$ ' y se multiplican por $0.65 R$. Para todos los modelos se tienen valores de $R=2$ en el modo fundamental, por lo que en todos los casos $0.65 R=1.3$.

- Adicionalmente, se revisan los casos de las acciones considerando los momentos flectores resistentes esperados de los elementos de la planta baja y las fuerzas cortantes asociadas a estos.

Los resultados se presentan para cada una de las columnas de planta baja. Las columnas se identifican de acuerdo con su posición en planta respecto a la dirección de aplicación de la carga en el AENL (figura 4). Los resultados de los análisis modales utilizando los diferentes espectros de respuesta consideran las diversas combinaciones de carga respecto a las NTC-04 y NTC-17. Para el caso de los resultados de los AENL, la carga se supone aplicada de manera monótona creciente en la dirección mostrada en la figura 4. Para tener resultados comparables con los de los análisis modales espectrales, se utilizan los valores máximos del AENL aplicado en ambos sentidos. 


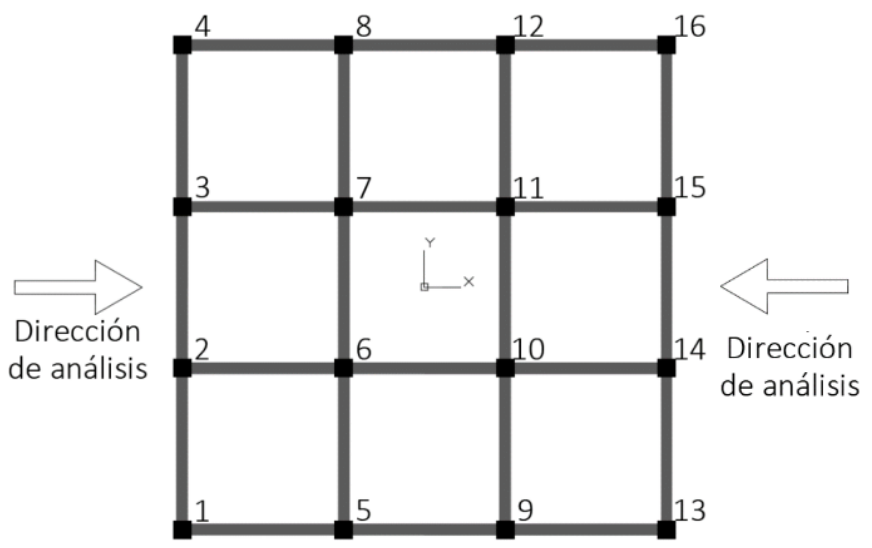

Figura 4. Identificación de columnas en planta respecto a la dirección de aplicación de la carga en el AENL
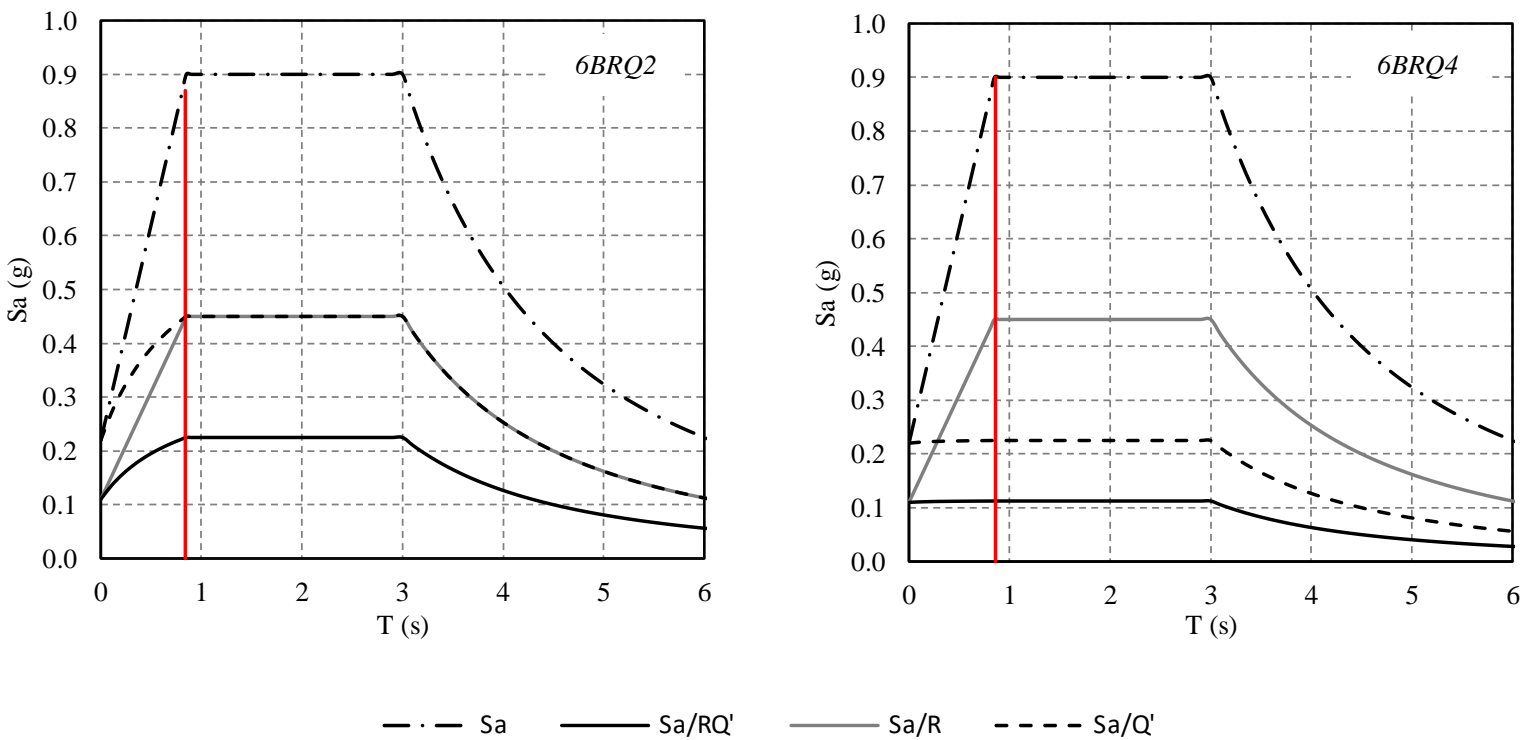

Figura 5. Espectros de diseño para los modelos de seis niveles (6BRQ2 y 6BRQ4) considerando las diferentes propuestas de combinación

En la figura 5 se muestran los espectros de diseño modificados descritos previamente para los modelos de seis niveles (6BRQ2 y 6BRQ4). Se indica con línea roja el periodo fundamental de vibrar de cada uno de los modelos. Se puede observar que para el modelo 6BRQ2, a partir del periodo correspondiente al inicio de la meseta, los espectros $S a / Q^{\prime}$ y $S a / R$ son idénticos, dado que a partir de este periodo los valores de $Q^{\prime}$ y $R$ son iguales de acuerdo con las NTC-04 para este caso. Dado que el periodo fundamental del modelo 6BRQ2 coincide con el inicio de la meseta, es de esperarse que las fuerzas producidas en ambos casos sean muy similares para este modelo. Las diferencias entre los resultados de ambos casos se asocian a la contribución de modos superiores que corresponden a la rama ascendente en ambos espectros. Para el modelo 6BRQ4, los cuatro espectros son claramente distintos, debido a las diferencias en los valores de $Q$, y $R$. Los valores de pseudoaceleración correspondientes al periodo fundamental del modelo del caso elástico son ocho veces mayores que los valores de diseño, como era de esperarse. 

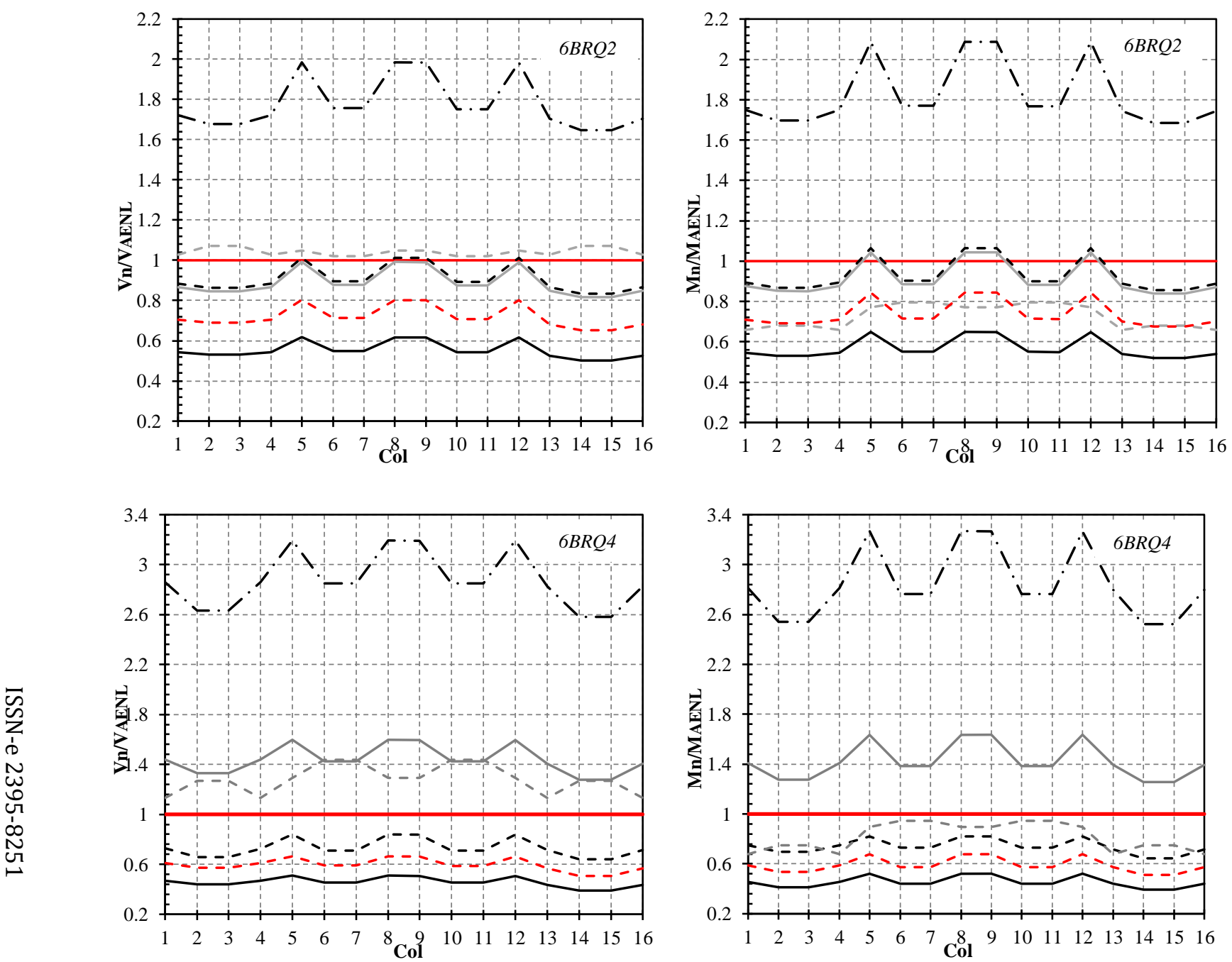

AENL

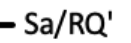

- - Sa

Sa /

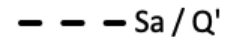

$--\mathrm{VR}$

NTC-17

Figura 6. Fuerzas cortantes $\left(\mathrm{V}_{\mathrm{n}} / \mathrm{V}_{\mathrm{AENL}}\right)$ y momentos normalizados $\left(\mathrm{M}_{\mathrm{n}} / \mathrm{M}_{\mathrm{AENL}}\right)$ respecto al AENL en la base de las columnas de planta baja para los modelos 6BRQ2 y 6BRQ4

En la figura 6 se muestran, para los modelos 6BRQ2 y 6BRQ4, las fuerzas cortantes y los momentos flectores en la base de las columnas de planta baja para cada propuesta de análisis ( $\mathrm{V}_{\mathrm{n}}$ y $\mathrm{M}_{\mathrm{n}}$ ) normalizados respecto a los valores obtenidos del AENL $\left(\mathrm{V}_{\mathrm{AENL}} \mathrm{y} \mathrm{M}_{\mathrm{AENL}}\right)$. Con línea roja continua se muestra el umbral de valores unitarios. Los valores por arriba de este umbral significan que la fuerza interna calculada con la propuesta de análisis es mayor que la obtenida con el AENL y viceversa. Se puede observar para ambos modelos que las distribuciones de fuerzas cortantes y momentos flectores obtenidas con los análisis modales espectrales no son iguales que las obtenidas del AENL. En cada una de las columnas, los cocientes entre cada uno de los análisis modales y el AENL son diferentes. En todos los casos, los elementos mecánicos obtenidos con los espectros de diseño reducidos son significativamente menores que los obtenidos de los AENL. Para el modelo 6BRQ2, los valores oscilan alrededor de 0.6 , lo que quiere decir que los elementos mecánicos obtenidos de esta manera son apenas el 60\% de los obtenidos del AENL. Para el modelo 6BRQ4, estos valores se reducen a valores cercanos a 0.45. Esta propuesta no considera la sobreresistencia del sistema estructural y es la que se incluía en las NTC04 y en muchos otros cuerpos normativos. Una situación similar se presenta cuando se consideran estos resultados 
multiplicados por $0.65 R$ de acuerdo con lo estipulado en las NTC-17, en donde se puede observar que utilizar solamente el $65 \%$ de la sobrerresistencia para incrementar las fuerzas de diseño no alcanza a cubrir los resultados de los AENL. De hecho, en algunos de los códigos se considera que si el sistema no se diseña como un sistema de alta ductilidad, la sobreresistencia de la superestructura no debe ser considerada en los elementos mecánicos de la cimentación.

Por otra parte, los elementos mecánicos obtenidos, considerando el espectro elástico, son mucho mayores, con valores normalizados de entre 1.7 y 2.1 para el modelo 6BRQ2 y de entre 2.6 y 3.4 para el modelo 6BRQ4. Estos resultados muestran que, para estos dos modelos, utilizar los elementos mecánicos considerando comportamiento elástico de la superestructura sería sumamente conservador. En cuanto a los resultados utilizando diseño por capacidad, considerando los momentos resistentes en las columnas y las fuerzas cortantes asociadas a los mismos, se observan diferencias entre los resultados para los momentos flectores y para las fuerzas cortantes. Los momentos resistentes se calcularon considerando la carga axial máxima de los análisis modales de diseño. Dada la dependencia de la resistencia a flexión de las columnas con la carga axial, existen condiciones en las cuales los momentos que se desarrollan en el AENL son mayores que los momentos resistentes. Los valores para estos casos oscilan entre 0.7 y 0.8 para el modelo 6BRQ2 y entre 0.7 y casi 1 para el modelo 6BRQ4. Es obvio que las columnas de los marcos externos respecto a la aplicación de la carga presentan mayores variaciones que las columnas centrales. En cuanto a los resultados de las fuerzas cortantes, puede observarse que en general los valores son mayores a 1 para ambos modelos.

En cuanto a los resultados obtenidos con el espectro reducido únicamente por sobrerresistencia $(\mathrm{Sa} / \mathrm{R})$, puede observarse que para el modelo 6BRQ2 los valores oscilan entre 0.8 y muy cercanos a la unidad, superando el valor de uno para los momentos flectores en algunas columnas. Sin embargo, en el modelo 6BRQ4, los valores son siempre mayores a la unidad, oscilando entre valores de 1.25 y 1.65 . Estos resultados reflejan que los elementos mecánicos calculados con esta propuesta sobreestiman los elementos mecánicos esperados para este modelo. La propuesta de utilizar un espectro reducido solamente por ductilidad $\left(S a / Q^{\prime}\right)$, la cuál es equivalente a tomar los elementos mecánicos de diseño y multiplicarlos por la sobreresistencia, produce resultados muy similares a $S a / R$ para el modelo 6BRQ2, con valores que oscilan entre 0.86 y 1 . Esta situación es consistente con lo destacado en los espectros de diseño de la figura 5. Por otro lado, en el modelo 6BRQ4, los resultados con esta propuesta siguen siendo menores a la unidad, con valores que oscilan entre 0.67 y 0.84 , lo cual se traduce en diferencias entre el $33 \%$ y el $16 \%$ respecto al AENL.

Una de las variables que pueden modificar los elementos mecánicos máximos que la superestructura puede desarrollaren su incursión en el comportamiento inelástico son los efectos de interacción suelo estructura. Como se mostrará en los modelos de diez niveles, los efectos de interacción suelo estructura modifican no sólo el comportamiento del sistema, sino que adicionalmente modifican los parámetros fundamentales de diseño y por lo tanto las demandas a las que están sometidos. Para estudiar exclusivamente la influencia de los efectos ISE en el comportamiento de los modelos, se realizó una comparación de los elementos mecánicos obtenidos mediante AENL de los modelos de seis niveles con base rígida y con base flexible. Los modelos de seis niveles con base flexible no se estudian a profundidad dado que no se realizó un análisis y diseño completo de los mismos. Estos modelos son exactamente iguales a los modelos 6BRQ2 y 6BEQ4 con la única diferencia de que en el AENL, en dichos modelos se ha incluido la influencia de la flexibilidad de la base mediante el uso de las funciones de impedancia, de forma similar que para el caso de los modelos 10BFQ2 y 10BFQ4. En la figura 7 se muestran los cocientes de los elementos mecánicos en la base de las columnas de los modelos con base flexible ( $\mathrm{V}_{\text {ISE }}$ y $\left.\mathrm{M}_{\text {ISE }}\right)$ y de los modelos con base rígida $\left(\mathrm{V}_{\mathrm{BR}}\right.$ y $\left.\mathrm{M}_{\mathrm{BR}}\right)$ para los diseños con $Q=2$ y $Q=4$. 

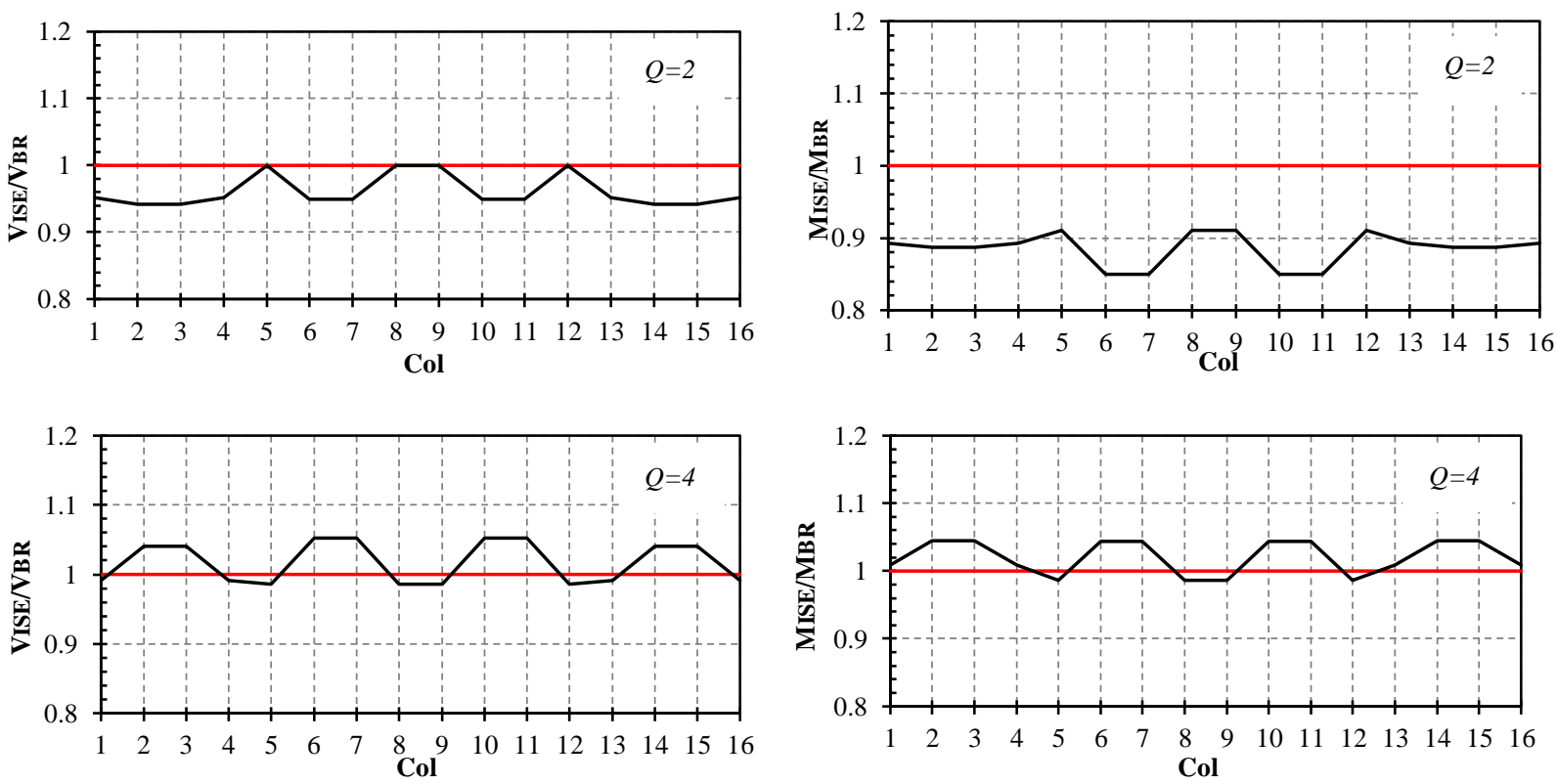

Figura 7. Cociente entre las fuerzas cortantes (VISE/VBR) y momento flector (MISE/MBR) en la base de las columnas de planta baja calculados con el AENL para los modelos con base flexible (ISE) y con base rígida (BR) del edificio de 6 niveles

De los resultados de la figura 7 se puede ver que para el modelo diseñado con $Q=2$, la flexibilidad de la base produce una reducción en las fuerzas cortantes en casi todas las columnas de menos del 10\% y una reducción en los momentos flectores de entre el 10 y el 15\%. Sin embargo, para el modelo $\mathrm{Q}=4$ la flexibilidad de la base produce un incremento en los elementos mecánicos de ocho columnas, tanto de fuerza cortante como de momento flector. Es importante destacar que estos resultados están asociados con la resistencia del sistema estructural y no con las demandas sobre el mismo. El efecto de la demanda se mostrará para los modelos de diez niveles. En todos los casos, los incrementos o reducciones son menores del $15 \%$.

Los espectros de diseño para las diferentes propuestas se muestran en la figura 8 para los modelos 10BRQ2 y 10BFQ2. De igual forma se indican con línea roja la posición de los periodos fundamentales de vibrar de ambos modelos. Para el caso del modelo sin efectos ISE (10BRQ2), las diferencias entre los espectros son similares que para el modelo de seis niveles (6BRQ2). El espectro elástico es alrededor de cuatro veces el espectro de diseño reducido por comportamiento inelástico y por sobreresistencia. Los espectros $S a / R$ y $S a / Q$ ' nuevamente son similares a partir del inicio de la meseta, debido a que los valores de $Q$ ' y $R$ son similares para este intervalo de periodos. Debido a que el periodo fundamental del modelo 10BRQ2 coincide con la meseta, las fuerzas a las que está sometido el modelo ante estos dos espectros son muy similares. En el modelo con efectos ISE (10BFQ2), los espectros de diseño sufren algunas modificaciones. En primer lugar, el espectro de respuesta elástico corresponde a ordenadas espectrales menores respecto al modelo sin efectos ISE. Esto obedece a que los efectos ISE producen un incremento en el amortiguamiento del sistema, como se reporta en la tabla 2. Se puede observar que los resultados de los espectros de diseño $S a / R$ y $S a / Q$ ' son diferentes. Esta diferencia surge de los valores reducidos de $Q$ ' que deben ser utilizados para los sistemas con efectos de ISE. De hecho, la necesidad de utilizar un valor de $Q$ ' reducido por efectos de ISE produce que la meseta del espectro de diseño inelásticos del modelo 10BFQ2 tenga valores de aceleración mayores que el del modelo 10BRQ2, aun cuando el espectro inelástico del segundo corresponde a ordenadas espectrales mayores. Este efecto muestra que, en muchos casos, los modelos con efectos ISE están sometidos a fuerzas mayores que los modelos con base rígida. Se puede observar que las fuerzas asociadas al periodo fundamental de este modelo son diferentes para las distintas 
propuestas, teniendo mayor fuerza para el espectro elástico $S a$, seguido por el espectro $S a / Q^{\prime}$, por el espectro $S a / R$ y finalmente por el espectro de diseño inelástico $S a / R Q$ '.
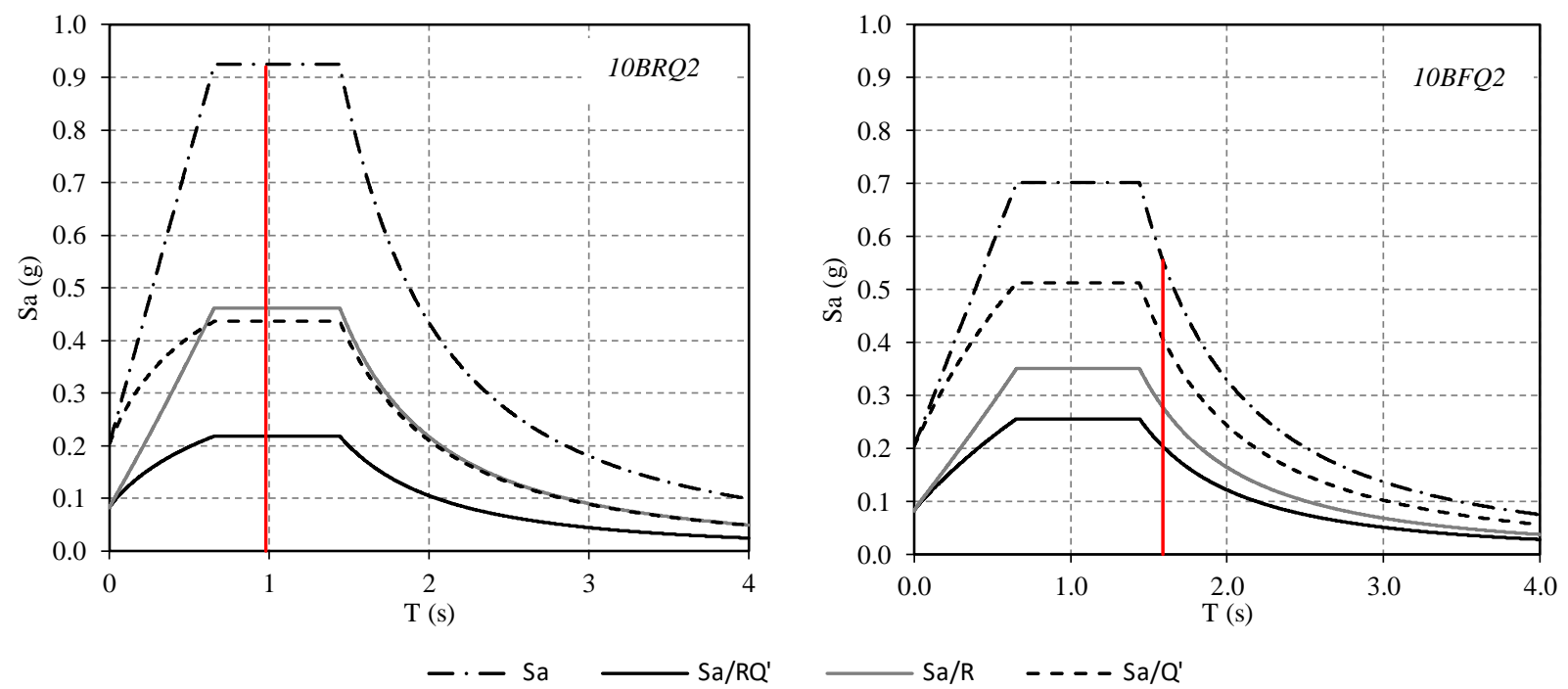

Figura 8. Espectros de diseño para los modelos de diez niveles con $Q=2$ con base rígida (BR) y con base flexible (BF), considerando las diferentes propuestas de combinación

Los resultados de los elementos mecánicos para las columnas de planta baja en los modelos 10BRQ2 y 10BFQ2 se muestran en la figura 9. En el modelo sin efectos de interacción (10BRQ2) se observan resultados similares que para el caso de seis niveles. Nuevamente los elementos mecánicos calculados con el espectro elástico oscilan entre 1.8 y 2. Así mismo, los elementos mecánicos calculados con el espectro de diseño reducido tienen valores mucho menores a la unidad. En este caso oscilan entre 0.4 y 0.5 tanto para las fuerzas cortante como para los momentos flectores. Para el criterio de las NTC-17 los resultados vuelven a ser menores a la unidad oscilando entre 0.5 y 0.65 para fuerzas cortantes y momentos flectores. Los resultados de las propuestas $S a / R$ y $S a / Q$ ' son muy similares, con valores menores a la unidad y mayores a 0.8 . En este caso es interesante que el valor de los momentos flectores resistentes es muy parecido a las propuestas de utilizar $S a / R$ y $S a / Q$ '. Sin embargo, en el caso de las fuerzas cortantes asociadas a los momentos resistentes, los valores oscilan alrededor de 1.6, mucho mayores que para el modelo de seis niveles. Para el modelo con efectos ISE (10BFQ2) los resultados se modifican notablemente. En primer lugar, los valores para el espectro elástico se ven reducidos, oscilando entre valores de 1.1 y 1.4. Este resultado se debe a que, en el sistema con base flexible, las reducciones por comportamiento inelástico son menores debido a los valores reducidos de $Q^{\prime}$ que se deben utilizar (tabla 2). En cuanto a los resultados de los espectros $S a / R$ y $S a / Q$ ', estos dejan de ser similares. Para el caso del espectro $S a / R$ los valores oscilan entre 0.55 y 0.7 , mientras que los resultados con el espectro $S a / Q$ ' son más cercanos a la unidad con valores que oscilan entre 0.8 y 1 . En términos de los momentos flectores resistentes y las fuerzas cortantes asociadas, los resultados son muy similares al modelo con base rígida. Las secciones y armados de las columnas de planta baja son iguales para ambos casos (tabla 1), debido a que la aceleración espectral en el modo fundamental para ambos modelos es muy parecida (figura 8). Esta es una situación particular de este modelo y en ninguna circunstancia debe extrapolarse a otros casos. 

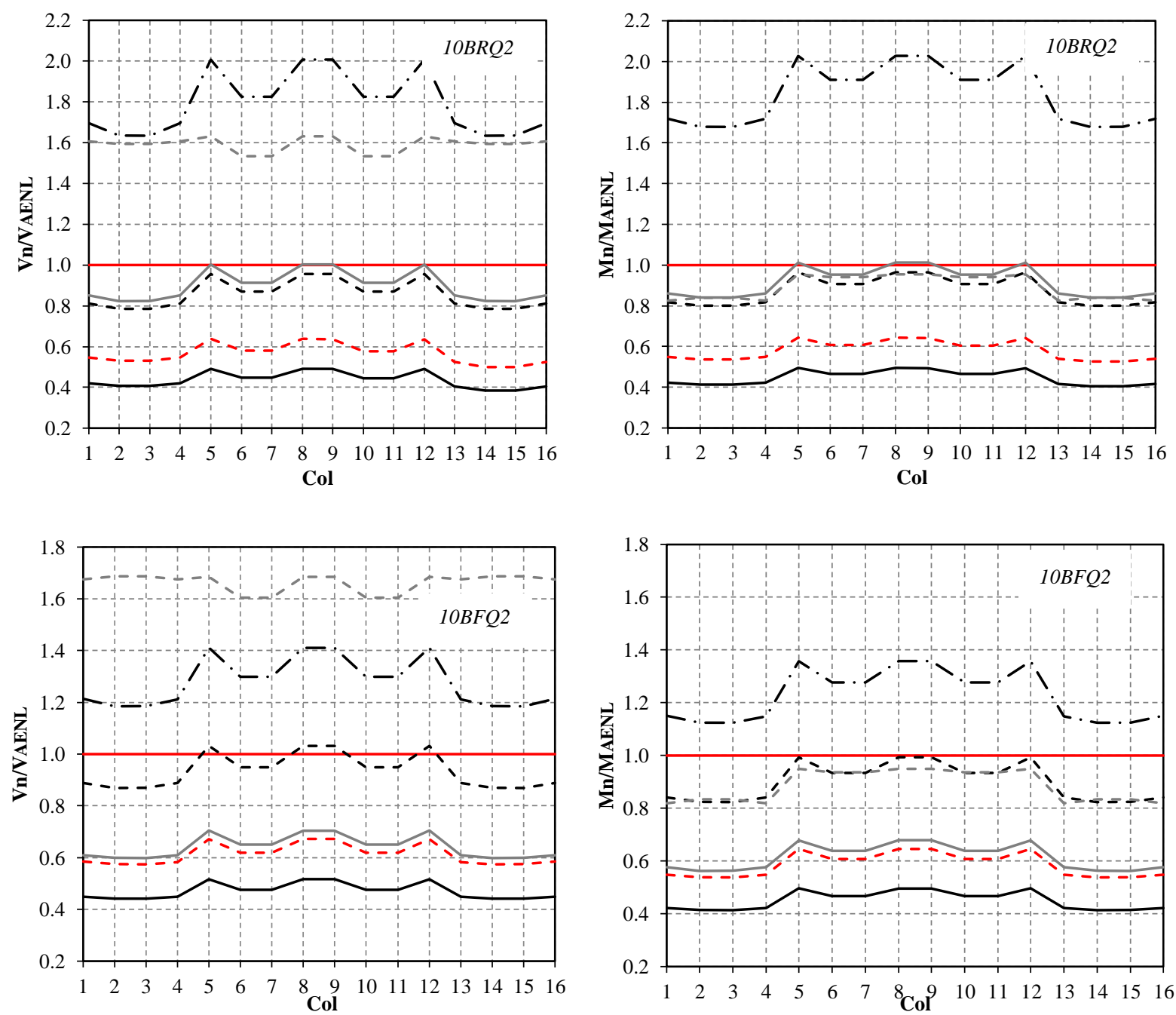

AENL $\mathrm{Sa} / \mathrm{RQ}^{\prime}$

Sa / R

- - - Sa / Q' - - VR - - - NTC-17

Figura 9. Fuerzas cortantes (Vn/VAENL) y momentos normalizados (Mn/MAENL) respecto al AENL en la base de las columnas de planta baja para los modelos $10 B R Q 2$ y $10 B F Q 2$

En la figura 10 se muestran los espectros de diseño de las distintas propuestas para los modelos diseñados con alta ductilidad sin y con efectos ISE (10BRQ4 y 10BFQ4). Los resultados del modelo con base rígida son similares a los del caso de seis niveles. De igual forma, los efectos sobre los espectros producidos por la ISE son similares a los del modelo 10BFQ2. El espectro elástico tiene ordenadas espectrales menores que el de base rígida, debido al incremento en el amortiguamiento del sistema (tabla 2). Como puede verse, el orden de los espectros, del mayor al menor, se ve invertido por los efectos ISE. En el caso de base rígida, las ordenadas del espectro $S a / R$ son mayores que las del espectro $S a / Q^{\prime}$, mientras que para el modelo con efectos ISE esta situación se invierte. Adicionalmente, es posible ver que el periodo fundamental del modelo 10BRQ4 corresponde a la meseta del espectro, mientras que los efectos ISE producen que el periodo fundamental del modelo 10BFQ4 corresponda a la rama descendente. 

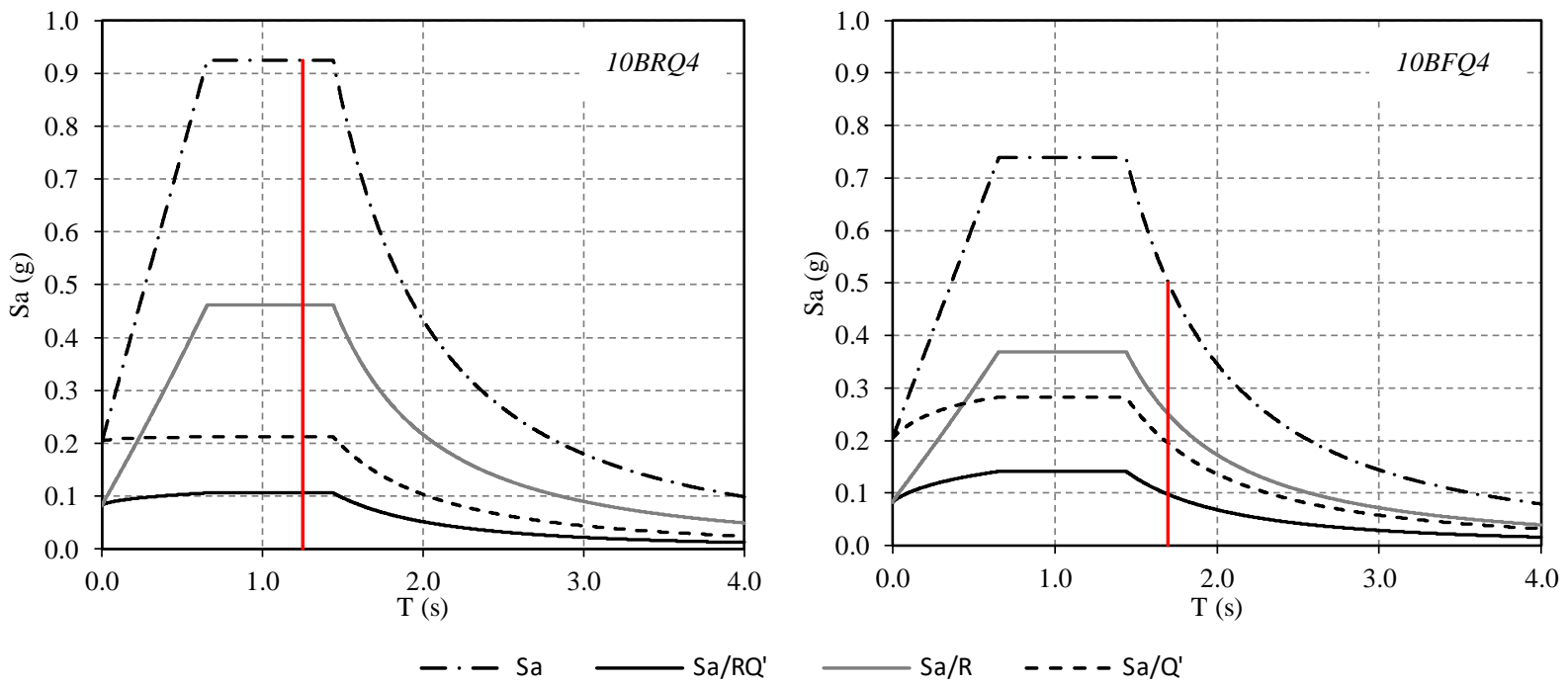

Figura 10. Espectros de diseño para los modelos de diez niveles con $Q=4$ con base rígida (BR) y con base flexible (BF), considerando las diferentes propuestas de combinación

Para los modelos 10BRQ4 y 10BFQ4, los elementos mecánicos normalizados se muestran en la figura 11. Los resultados con el espectro elástico del modelo con base rígida oscilan entre 2.8 y 3.4 para las fuerzas cortantes y entre 3 y 3.6 para los momentos flectores. Para el caso de los elementos mecánicos de diseño con el espectro reducido se tienen valores menores a $0.4,60 \%$ menores que los elementos mecánicos calculados con el AENL mientras que para el criterio de las NTC-17 los valores son 50\% menores. Para este modelo, los valores calculados con el espectro $S a / R$ oscilan entre 1.4 y 1.8 tanto para fuerzas cortantes como para momentos flectores, mientras que con el espectro $S a / Q$ ' se obtienen valores entre 0.67 y 0.85 , iguales que para el modelo de seis niveles. Los momentos resistentes en este modelo tienen valores cercanos a la unidad, mostrando un alto grado de aproximación, sin embargo, las fuerzas cortantes asociadas a estos momentos oscilan alrededor de 1.4. En cuanto al modelo que incluye efectos ISE, los valores calculados con el espectro elástico son de entre 1.8 y 2 para las fuerzas cortantes y entre 1.8 y 2.2 para los momentos flectores. Esta reducción en los valores respecto a los de base rígida, nuevamente están asociados con los valores reducidos de $Q$ ' por efectos ISE (tabla 2). En este caso, los valores calculados con el espectro $S a / R$ son muy cercanos a la unidad tanto para fuerzas cortantes como para momentos flectores. Por otra parte, los valores calculados con el espectro $\mathrm{Sa} / \mathrm{Q}$ ' son menores a uno con valores mínimos de 0.7 para algunas de las columnas. Al igual que para el modelo 10BRQ4, los momentos flectores resistentes son muy parecidos a los resultados del AENL, con valores de fuerzas cortantes asociadas de entre 1.4 y 1.5.

Al igual que para los modelos de seis niveles, se realizó la comparación de los elementos mecánicos calculados de los AENL con base flexible y con base rígida. El cociente de los resultados de base flexible ( $\mathrm{V}_{\mathrm{ISE}}$ y $\mathrm{M}_{\mathrm{ISE}}$ ) entre los de base rígida ( $\mathrm{V}_{\mathrm{BR}}$ y $\mathrm{M}_{\mathrm{BR}}$ ) se muestra en la figura 12. En este caso, los momentos flectores para ambos modelos son muy similares, mientras que las fuerzas cortantes en el modelo diseñado con $\mathrm{Q}=2$ se ven ligeramente reducidas por la flexibilidad de la base. Para el caso con $\mathrm{Q}=4$, las fuerzas cortantes también son similares. No es de sorprender que los efectos ISE en los modelos de diez niveles sean un poco menores que para los modelos de seis niveles, dado que en general, a medida que las estructuras son más flexibles, los efectos ISE suelen ser menores. 

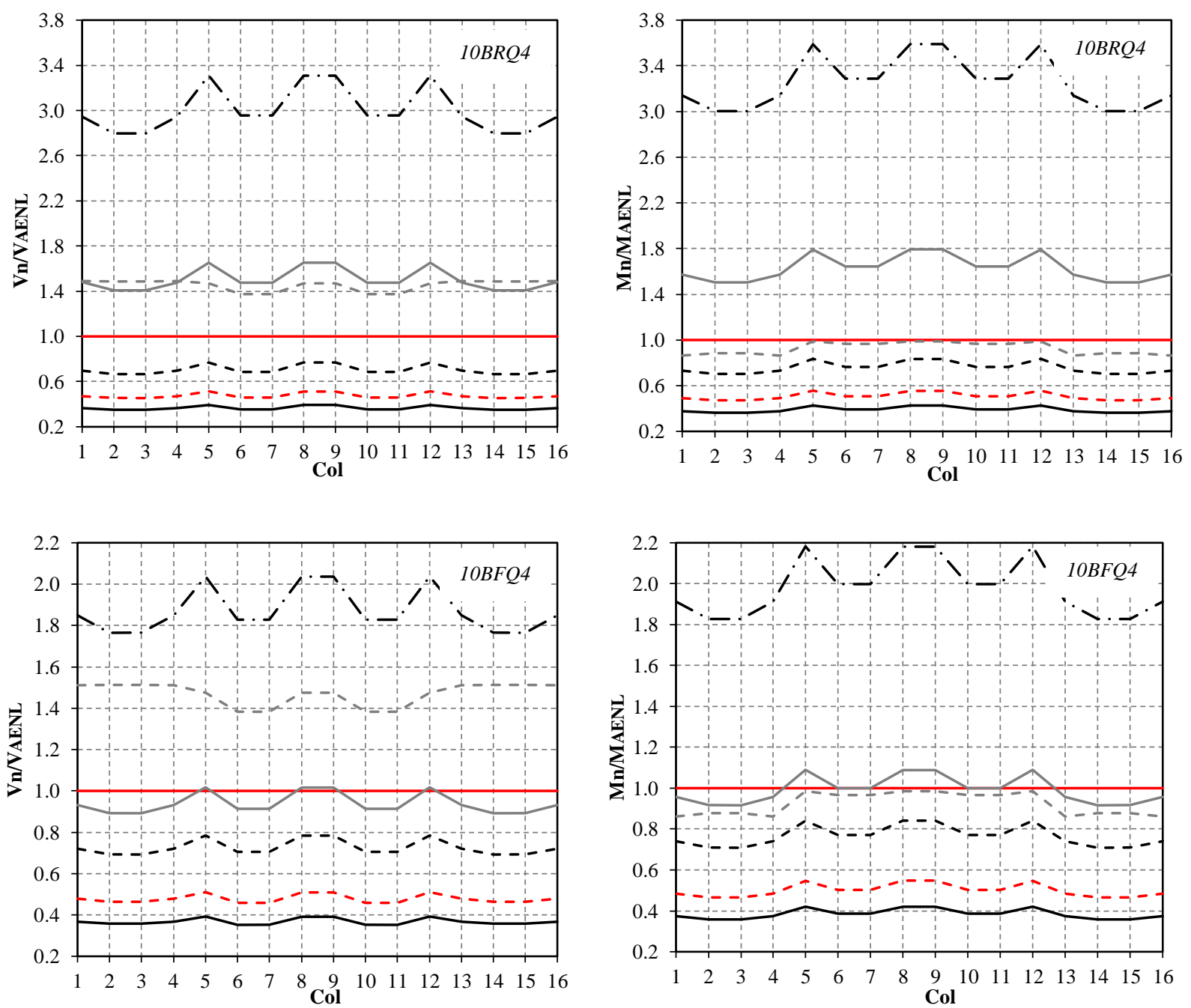

AENL $\mathrm{Sa} / \mathrm{RQ}^{\prime}$

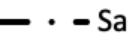

Sa /

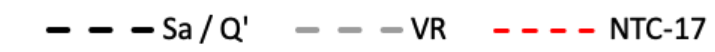

Figura 11. Fuerzas cortantes (Vn/VAENL) y momentos normalizados (Mn/MAENL) respecto al AENL en la base de las columnas de planta baja para los modelos $10 B R Q 4$ y $10 B F Q 4$

\section{CONCLUSIONES}

Se presenta un estudio acerca de las acciones máximas de diseño que se deben de utilizar para cimentaciones de estructuras en las cuales se desea que se desarrollen mecanismos de comportamiento inelástico estables. Considerando los principios de diseño por capacidad, es necesario que el sistema suelocimentación sea capaz de resistir las máximas acciones que se produzcan en la superestructura cuando ésta desarrolla por completo su capacidad de deformación inelástica. En el diseño convencional de cimentaciones ante acciones sísmicas se suelen utilizar los elementos mecánicos calculados bajo la acción de espectros de diseño reducidos por comportamiento inelástico y sobre-resistencia. Sin embargo, las acciones a las que puede estar sometida la cimentación son mayores debido a la sobre-resistencia que presentan los sistemas estructurales hiperestáticos. 

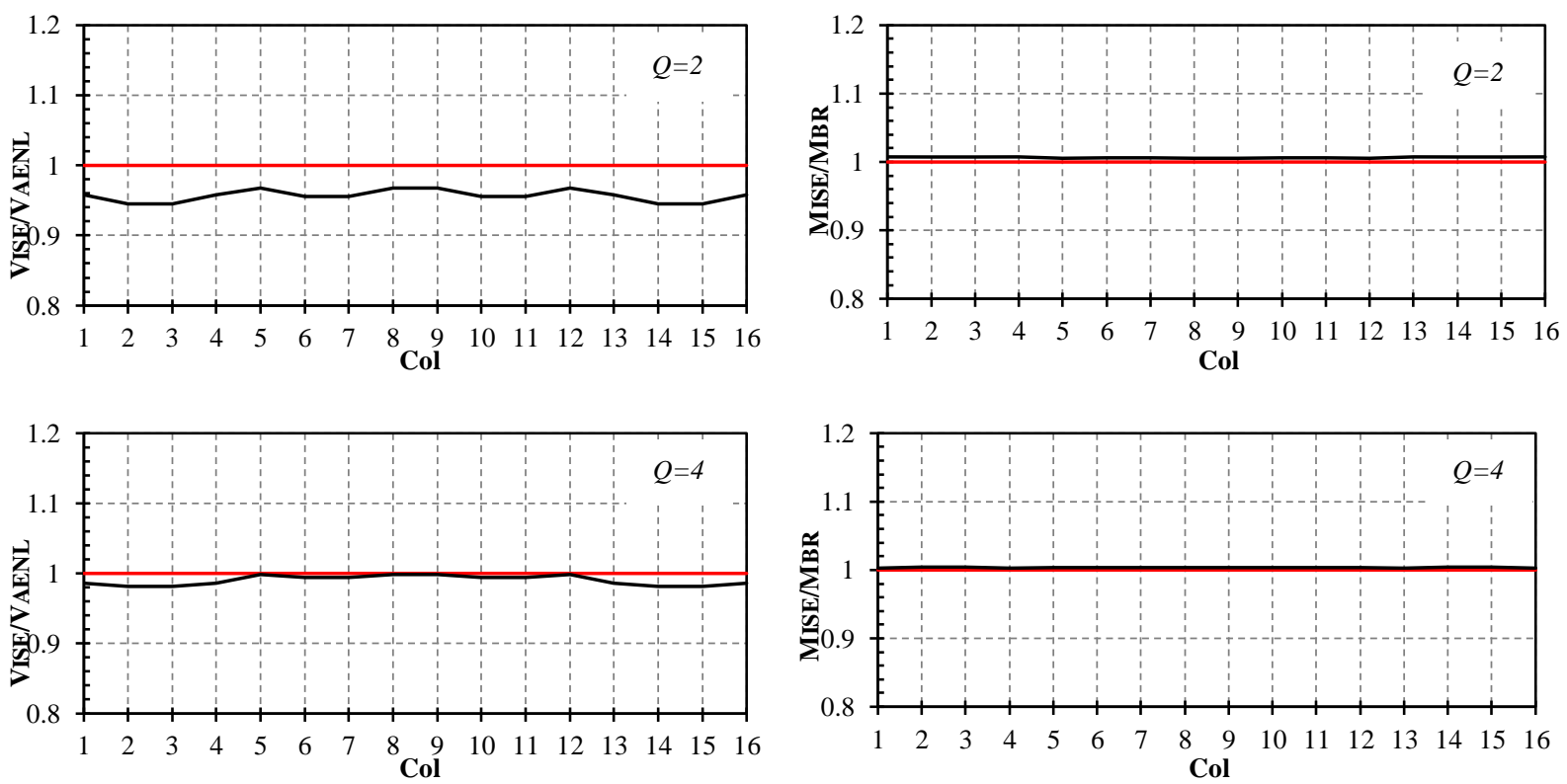

Figura 12. Cociente entre las fuerzas cortantes (VISE/VBR) y momento flector (MISE/MBR) en la base de las columnas de planta baja calculados con el AENL para los modelos con base flexible (ISE) y con base rígida $(\mathrm{BR})$ del edificio de 10 niveles

Dada la necesidad de proveer a los diseñadores estructurales de metodologías sencillas para determinar las acciones de diseño en las cimentaciones, se presentan tres propuestas de análisis basadas en espectros de diseño modificados para su fácil implementación. Se comparan los elementos mecánicos (fuerza cortante y momento flector) en la base de las columnas de planta baja obtenidos con: el espectro de diseño elástico; el espectro de diseño reducido solamente por el factor de sobre-resistencia (equivalente a los espectros elásticos del cuerpo principal de las NTC-04); el espectro de diseño reducido exclusivamente por comportamiento inelástico (equivalente a tomar los resultados obtenidos de los espectros de diseño reducidos y multiplicarlos por el factor de sobre-resistencia, como lo plantean algunos reglamentos); y los del espectro de diseño reducido por comportamiento inelástico y sobre-resistencia (análisis convencional). También se comparan los resultados de aplicar el criterio incluido en las NTC-17. Adicionalmente se muestran la comparación con los momentos flectores resistentes y las fuerzas cortantes asociadas a los mismos (diseño por capacidad). Se utilizan los elementos mecánicos obtenidos de análisis estáticos no lineales (AENL) como referencia. Los resultados se presentan como el cociente entre los elementos mecánicos obtenidos de cada uno de los análisis y los obtenidos del AENL.

Se analizan edificios de concreto reforzado con marcos momento resistentes. Dos edificios de seis niveles diseñados con diferentes factores de comportamiento sísmico $(\mathrm{Q}=2$ y $Q=4)$. Además, cuatro edificios de diez niveles, dos sin considerar efectos de interacción suelo estructura (base rígida) y dos considerándolos (base flexible) con los mismos factores de comportamiento sísmico que los de seis niveles. Los edificios de seis niveles no se diseñaron con efectos de interacción suelo estructura, sin embargo, se analizaron considerando la base flexible para estudiar la influencia de este efecto en los resultados de los análisis estáticos no lineales.

En primera instancia se muestra que, en todos los casos, los elementos mecánicos calculados con los espectros de diseño reducidos (diseño convencional) son mucho menores que los obtenidos del AENL. Los valores normalizados oscilan alrededor de 0.4, lo cual quiere decir que las fuerzas determinadas corresponden solamente al $40 \%$ de las que se obtuvieron en el AENL. Esta subestimación de las fuerzas 
máxima no se logra corregir al multiplicarlos por $0.65 R$, tal como se indica en las NTC-17 y los resultados oscilan alrededor del $50 \%$ de las fuerzas determinadas con el AENL.

La totalidad de los casos estudiados presentan que tanto las fuerzas cortantes como los momentos flectores obtenidos con los espectros de respuesta elásticos son mayores que los del AENL. Para los edificios con base rígida y $\mathrm{Q}=2$ se obtienen elementos mecánicos normalizados con valores de entre 1.8 y 2 , mientras que para los modelos de $\mathrm{Q}=4$ los valores oscilan entre 3.2 y 3.6. En el caso de los modelos con base flexible, los valores se reducen entre 1.2 y 1.4 para $Q=2$ y entre 2 y 2.2 para $Q=4$.

Respecto a los resultados obtenidos con las resistencias de las columnas, se puede ver que existe una buena aproximación en términos de los momentos flectores. Sin embargo, las fuerzas cortantes asociadas son mucho mayores que las estimadas con los AENL en todos los casos. De hecho, para el modelo de diez niveles con base flexible y $\mathrm{Q}=2$, las fuerzas cortantes obtenidas de esta manera son incluso mayores que las obtenidas del análisis elástico.

Los resultados obtenidos con el espectro reducido exclusivamente por sobre-resistencia muestran poca consistencia. Mientras que en los modelos con base rígida diseñados para $\mathrm{Q}=2$ se tienen valores cercanos a la unidad, en el resto de los modelos los elementos mecánicos calculados con esta propuesta pueden ser mayores o menores respecto a los del AENL.

El uso de un espectro reducido solamente por comportamiento inelástico parece se la propuesta con mejor nivel de aproximación en todos los casos. En general los elementos mecánicos obtenidos de esta manera son ligeramente menores que los obtenidos con el AENL, con valores mínimos de 0.67 en el caso con mayor desviación. Se puede observar que estos valores se presentan principalmente en las columnas de los marcos perimetrales, debido a que en estas columnas las variaciones de la carga axial son las mayores, lo cual impacta directamente en la resistencia a flexión de la columna y por lo tanto en los elementos mecánicos que puede desarrollar.

En general los resultados de los análisis estáticos no lineales de los modelos con base rígida y con base flexible son similares. Sin embargo, aun cuando el diseño de las columnas de planta baja fue igual para ambos modelos, existen diferencias en algunas columnas. Para el modelo de seis niveles con $\mathrm{Q}=2$, el modelo con base flexible desarrolla elementos mecánicos menores mientras que para el modelo de $\mathrm{Q}=4$ sucede lo contrario. En los modelos de diez niveles los resultados entre ambos modelos son muy similares.

Los resultados obtenidos en este trabajo se presentan exclusivamente para estructuras regulares de marcos de concreto. Dado que la sobrerresistencia depende del tipo de material, la estructuración e incluso el periodo fundamental de vibrar de la estructura, es necesario estudiar las propuestas aquí descritas considerando estas variables. Adicionalmente es necesario corroborar los resultados utilizando análisis dinámicos incrementales para corroborar las tendencias identificadas en este trabajo.

\section{AGRADECIMIENTOS}

Este estudio ha sido patrocinado por el Instituto para la Seguridad de las Construcciones mediante los proyectos UAM-A/DMAE-2015/07, UAM-A/DMAE-2016/03 y UAM-A/DMAE-2017/10. Los autores agradecen al Consejo Nacional de Ciencia y Tecnología por las becas otorgadas a dos de los autores para desarrollar sus estudios de maestría. 


\section{REFERENCIAS}

Anastasopoulos, I, G Gazetas, M Loli, M Apostolou, y N Gerolymos (2010). "Soil failure can be used for seismic protection of structures". Bulletin of Earthquake Engineering, 8(2), pp. 309-326. DOI: $\underline{10.1007 / \mathrm{s} 10518-009-9145-2}$

Avilés, J y L E Pérez-Rocha (2003). "Soil-structure interaction in yielding systems". Earthquake engineering \& structural dynamics, 32(11), pp. 1749-1771. DOI: 10.1002/eqe.300

Burgos Namuche, M (2007). "Estudio de la metodología “diseño por capacidad” en edificaciones aporticadas de concreto armado para ser incorporada a la norma peruana E-060 como alternativa de diseño" Tesis Maestría, Universidad Nacional De Ingeniería, Facultad de Ingeniería Civil Sección de Posgrado, Lima Perú.

Castro Tello, C F (2005). "Estudio de una estructura de quince niveles a base de marcos de concreto reforzado para asegurar el mecanismo plástico columna fuerte-viga débil". Tesis Maestría, Universidad Nacional Autónoma de México, México D.F.

CSI (2017), "SAP2000 - Integrated Finite Elements Analysis and Design of Structures - CSI Analysis Reference Manual - Version 19”, Computers and Structures, Inc. (CSI), Berkeley, California, E.U.A.

Federal Emergency Management Agency (FEMA, P. 2000). "Commentary for the Seismic Rehabilitation of Buildings". FEMA-356, Federal Emergency Management Agency, Washington, DC.

Fernandez-Sola, L R y J E Huerta-Ecatl (2018). "Inelastic behavior of systems with flexible base". Earthquakes and Structures, 14(5), pp. 411-424. DOI: 10.12989/eas.2018.14.5.411

Gazetas, G (1983), "Analysis of machine foundation vibrations: state of the art", Soil Dynamics and Earthquake Engineering, Vol. 2, pp. 2-42. DOI: 10.1016/0261-7277(83)90025-6

Hernández-Rodríguez, L F (2016). "Acciones de diseño sobre la cimentación, para garantizar un adecuado comportamiento por capacidad en edificios de concreto reforzado". Tesis Maestría, Universidad Autónoma Metropolitana, México D.F.

Lin, P C, T Takeuchi y R Matsui (2019). "Optimal design of multiple damped-outrigger system incorporating buckling-restrained braces". Engineering Structures, 194, pp. 441-457. DOI: 10.1016/j.engstruct.2019.05.078

Loeza-Pérez, C E (2019). "Revisión de criterios para el diseño por capacidad de cimentaciones de edificios de concreto reforzado". Tesis Maestría, Universidad Autónoma Metropolitana, México D.F.

Nangullasmú, H y A Tena-Colunga (2017). Modelado de disipadores histeréticos de energía en estructuras de concreto reforzado. Diferencias entre análisis en el plano y en tres dimensiones. Revista Internacional de Ingeniería de Estructuras, 22(2), pp. 135-162.

NTC-04, (2004). Normas técnicas complementarias, Gaceta Oficial del Distrito Federal.

NTC-17, (2017). Normas técnicas complementarias, Gaceta Oficial del Distrito Federal.

Nuzzo, I, D Losanno y N Caterino (2019). "Seismic design and retrofit of frame structures with hysteretic dampers: a simplified displacement-based procedure". Bulletin of Earthquake Engineering, 17(5), 2787-2819. DOI: $10.1007 / \mathrm{s} 10518-019-00558-8$

O'Reilly, G J y G M Calvi (2019). "Conceptual seismic design in performance-based earthquake engineering”. Earthquake Engineering \& Structural Dynamics, 48(4), pp. 389-411. DOI: 10.1002/eqe.3141 
Piqué del Pozo, J (2002). "Diseño por capacidad: estrategia neozelandesa de diseño sismorresistente. En Microzonificación y su aplicación en la mitigación de desastres”, pp. 1-15. CISMID.

Palacios-Villalva, C (2005). "Análisis de un edificio de cinco niveles a base de marcos de concreto reforzado para asegurar el mecanismo plástico columna fuerte-viga débil”. Tesis Maestría, Universidad Nacional Autónoma de México, México D.F.

Rodríguez, M E y J C Botero (1997) "Evaluación del comportamiento de barras de acero de refuerzo sometidas a cargas monotónicas y cíclicas reversibles incluyendo pandeo" Revista de Ingeniería Sísmica, No. 56, pp. 9-27. DOI: 10.18867/RIS.56.230

Tapia Hernández, E y A Tena-Colunga (2013). "Diseño sísmico de marcos de acero contraventeados: Parte 1: recomendaciones de diseño". Revista de Ingeniería Sísmica, No. 88, pp. 43-68. DOI: 10.18867/RIS.88.9

Tena-Colunga, A y H Hernández (2018). "Diseño sísmico resiliente de marcos dúctiles de acero con fusibles estructurales”. Revista Internacional de Ingeniería de Estructuras, 22(4), pp. 421-457.

Wallace, J W (1992). "BIAX: revision 1, a computer program for the analysis of reinforced concrete sections". Clarkson University, Department of Civil Engineering. 